\title{
A Dynamic Channel Assignment in Low Earth Orbit Mobile Satellite Systems
}

\author{
Heesoo Lee, Sok-kyu Lee, and KyungHi Chang \\ Radio Transmission Technology Research Team, ETRI \\ Daejon 305-350, KOREA \\ e-mail: \{heelee, sk-lee, khchang\}@etri.re.kr
}

\author{
Sehun Kim \\ Industrial Engineering, KAIST \\ Daejon 305-701, KOREA \\ e-mail: shkim@kaist.ac.kr
}

\begin{abstract}
Several low earth orbit (LEO) satellite constellations have been proposed for mobile satellite systems (MSS's). Due to nonstationary coverage regions of individual LEO satellites, the offered traffic load for each cell varies continuously. The variation of the traffic load essentially requires dynamic channel assignment. Furthermore, extremely frequent handover requests require specific techniques that prioritize the service of handover requests. In this paper, we suggest a new dynamic channel assignment (DCA) scheme which eliminates forced call terminations due to handover failure (call dropping). This scheme utilizes frequency spectrum efficiently, and can also provide users a high-quality premium service that guarantees the success of each handover procedure, called guaranteed handover service. This is based on position measurement. Finally, we compare our algorithm with some other DCA algorithms by computational experiments. The results of experiments show that our algorithm provides systems higher throughput (higher completed call ratio) as well as guaranteed handovers.
\end{abstract}

Keywords-low earth orbit mobile satellite systems, dynamic channel assignment

\section{INTRODUCTION}

Several satellite orbital constellations have been proposed for MSS's. An interesting solution is given by LEO satellites, since they permit relaxation of the constraints on the link budget, allow the use of low-power handheld mobile terminals, and ensure the earth coverage with smaller cells, so achieving a higher traffic capacity. In any MSS, the geographical area served is divided into cells. Each cell is illuminated by a radiation spot-beam of a satellite. For LEO satellites, at relatively low altitude, the resulting cells have smaller size than the ones obtained by GEO satellites. LEO satellites move with respect to a fixed observer on the Earth surface. The velocity of a LEO satellite relative to a fixed observer is very fast. Because of this nonstationary characteristic, the coverage area of a LEO satellite changes continuously. Due to nonstationary coverage regions of individual satellites, the offered traffic load for each cell varies continuously. The variation of the traffic load essentially requires dynamic resource allocation. With respect to the frequency spectrum, dynamic channel assignment (DCA) scheme is necessary.

When an active mobile subscriber goes out from a cell and enters an adjacent one, a new channel must be automatically assigned to it in order to have a seamless conversation. This procedure is called handover; it involves the rerouting of a call between two adjacent beams that may belong to either the same satellite or two adjacent satellites of the MSS's. If no channel is available in the destination cell, the handover is unsuccessful and the call is dropped.

The selection of a suitable policy for managing handover requests is a central issue in defining resource management strategies. From the user standpoint, the interruption of a conversation is more undesirable than the blocking of a newly arriving call. In LEO-MSS's, interbeam handover requests are extremely frequent during call lifetime and at each beam change the call may be dropped due to an unsuccessful handover. Hence, LEO-MSS's require specific techniques that prioritize the service of handover requests with respect to the service of new call attempts in order to reduce as much as possible the call dropping probability.

For LEO-MSS, there are many previous researches dealing with DCA [1]-[3], and handover prioritization [4]-[10]. Moreover, a few researches dealt with handover prioritization under DCA in LEO-MSS [1], [2], [4], [6]. G. Maral et al. in [6] suggested a handover prioitization scheme under fixed channel assignment scheme in LEO-MSS, which provides near-to-zero call forced termination probability due to handover failure. This scheme can provide users a highquality premium service that guarantees the success of each handover procedure, called guaranteed handover service.

In this paper, we suggest a new DCA scheme which eliminates forced call terminations due to handover failure (call dropping). This is based on position measurement. The user terminals are assumed to be equipped with Global Positioning System (GPS) receivers, and hence the user locations are known. In addition to user terminal location information, our scheme uses the characteristic of an LEO that the mobility in the system can be approximated by the deterministic movement of the satellite travel with a constant speed. The user location information and deterministic mobility model provide us the future handover behavior of a user terminal.

\section{NEW DCA ALGORITHM}

\section{A. Assumptions}

LEO satellites are not stationary with respect to a fixed point on the earth: the satellite ground-track speed is far greater than the earth rotation speed and the user speed. 
Considering the high value of the satellite's orbital speed, we can neglect both the earth rotation speed and the user speed, i.e., the mobility in the system can be approximated by the deterministic movement of the satellite travel with a constant speed [1], [3]. Our DCA scheme uses the current position information of each user terminal. Recent developments in the positioning technology (e.g., GPS) and the ever-increasing public awareness of the necessity of vehicle emergency services provide strong assurance that accurate position measurements will become a commercially viable reality in the near future [5]. The technique of GPS has been gaining popularity at a fast pace. It has been widely used in military operations and many commercial applications related to the provision of road safety services, fleet tracking, and intelligent transportation systems. The accuracy achieved by GPS using basic point positioning technique is $100 \mathrm{~m}$ at the $95 \%$ probability level. If differential GPS is employed, accuracy at the $3-5 \mathrm{~m}$ level can be achieved.

In this paper, the user terminals are assumed to be equipped with GPS receivers, and hence the user locations are known. Moreover, the spot-beams of the LEO satellites move along known trajectories on the earth surface with a constant speed. Both the deterministic spot-beam movement and the user location information provide the future locations and the handover patterns of the user terminals.

\section{B. Algorithm Description}

The coverage area is divided into cells with each cell illuminated by an antenna spot-beam of a satellite. In the illustrated example, it is assumed that all the cells are hexagonal regular for simplicity as shown in Fig. 1, however, our DCA algorithm does not necessarily require hexagonal regular cellular layout.

Channels are assigned to cells in order to maintain the cochannel interference at an acceptable level. This means that the same channel can be used in different cells with centers distance larger than reuse distance $D$. With dynamic channel allocation, all channels are kept in a common pool, from which any channel can be allocated to any cell, as long as the reuse distance is guaranteed (or a certain signal quality can be maintained). In DCA schemes, the total satellite capacity can be variably shared by all beams. In centralized schemes, a channel from the central common pool is assign to a call by a centralized controller, which may reside in the satellite. In the Distributed DCA schemes, which are especially suitable for microcellular systems, base stations and users within a radio cell are responsible for the channel allocation in that cell. In this paper, we concentrate on the centralized DCA schemes.

In this section, our DCA algorithm is introduced with an example depicted in Fig. 1. Twenty-four spot-beams are moving rightward as a result of the satellite movement. Fig. 1 presents six snapshots taken in the time interval $\left[t_{0}, t_{5}\right]$. The solid lines show the current positions of the spot-beams while the dashed lines show the initial positions of the spot-beams. At time $t=t_{0}$ (configuration a), a user terminal, which is denoted as "user 0 " and is located in spot-beam $M$, requires a connection to be set up. It is assumed that the reuse distance $D=3 R^{\prime}$ in this example, where $R^{\prime}$ is the cell radius.
In general DCA schemes, the call setup request of the user 0 is accepted, if the cell $M$ has an available channel. Both the deterministic spot-beam movement and the user location information make us be able to forecast when the future handovers of the user terminals in the LEO system occur. Therefore, if the call setup request is accepted, we can expect that user 0 resides in the spot-beam $M$ until the handover instant at the time $t=t_{4}$ (configuration e).

Assume that the call setup request of the user 0 is accepted at the time $t=t_{0}$. If a handover request arrives by the user 0 , first we liberate the channel used in the origin cell $M$ the call originates from, and then search an available channel in the transit cell $L$ the call enters in. If there are available channels in the transit cell, an available channel is assigned to the handover call by a certain criteria used in the DCA scheme. Otherwise, the handover call is dropped. Defining the set of interfering cells of cell $x, I(x)$, as the set of cells distant less than $D$ from cell $x$, then the sets of interfering cells of cell $M$ and $L$ are $I(M)=\{G, H, L, N, R, S\} \quad, \quad$ and $I(L)=\{F, G, K, M, Q, R\}$ respectively. Assume that the user 0 requests a handover at the time $t=t_{4}$. It is sure that any user terminals in the cells of $I(M)$ isn't using the channel the user 0 has used in the origin cell at the time $t=t_{4}$. Since the two sets, $I(M)$ and $I(L)$, have a lot of common interfering cells, it is probable that the channel is not allocated to any of the cells in the set $I(L)$ at the time $t=t_{4}$. If this is true, the channel can be assigned to the user 0 in the transit cell at the time $t=t_{4}$, that is, the channel can remain associate with the user 0 . However, it is not always possible. In other words, it is also probable that the channel is already allocated to a certain cell in the set $I(L)-I(M)$ at the time $t=t_{4}$.

If it is guaranteed that the channel the handover request terminal has used in the origin cell is not allocated to any of the cells in the set of interfering cells of the transit cell at the handover instant, we can provide users zero call forced termination probability. By using both the deterministic spotbeam movement and the user location information, we can assign to a new call such a channel that can be used in the transit cell that the call will enter in at the future handover instant, and hence guarantee the success of each handover to all users. We show how this can be done with an example depicted in Fig. 1.

At time $t=t_{0}$ (configuration a), a user terminal, which is denoted as "user 0 " and is located in spot-beam $M$, requires a connection to be set up. The small black circles represent some of the existing user terminals at the time $t=t_{0}$, labeled as 1, 2, 3, and 4. Assume that the user $i$ is using the channel $C_{i}$, and $C_{i} \neq C_{j}$ for all $i \neq j$, and there are only four available channels for the cell $M$. If the system uses a conventional DCA scheme like the first available scheme or the nearest neighbor scheme, we can choose a channel randomly from the channel pool and assign it to the user 0 . However, our DCA algorithm chooses the channel $C_{3}$ to guarantee successful handovers in future to all users. From now on, we show how the channel $C_{3}$ can guarantee successful handovers.

Assume that the channel $C_{1}$ is assigned to the user 0 at the time $t=t_{0}$, when the call setup request by the user 0 arrives. 
At the time $t=t_{1}$ (configuration $\mathrm{b}$ ), the user 1 handovers to spot-beam $G$, and the user 0 resides in the cell $M$, using the channel $C_{1}$. Since the two cell $G$ and $M$ are distant less than the reuse distance from each other, the channel $C_{1}$ can not remain associate with the user 1 , if the user 0 is still active at the time $t=t_{1}$, that is, the user 1 should be assigned an channel different from the channel $C_{1}$. If there is no channel available for the cell $G$ at the time $t=t_{1}$, the user $l$ is forced to terminate. Therefore, we cannot guarantee a successful handover to the user 1 .

Let's examine the case that the channel $C_{2}$ is assigned to the user 0 at the time $t=t_{0}$. This is similar to the former case that the channel $C_{1}$ is assigned to the user 0 . At the time $t=t_{2}$ (configuration c), the user 2 handovers to spot-beam $S$. According to the same reason as for the former case, we cannot guarantee a successful handover to the user 2 .

Assume that the channel $C_{1}$ is assigned to the user 0 . At the time $t=t_{3}$ (configuration d), the user 3 handovers to spotbeam $J$. Assume that the channel is available for the spotbeam $J$ at the time $t=t_{3}$, and remain associate with the user 3 in the transit spot-beam $J$. At the time $t=t_{4}$ (Configuration e), the user 0 handovers to spot-beam $L$. At this time, the user 3 resides in the cell $J$, using the channel $C_{3}$. Since the two cell $J$ and $L$ is distant more than the reuse distance from each other, the channel $C_{3}$ can remain associate with the user 0 , even though the user 3 is still active. Therefore, the handover request at the time $t=t_{4}$ can be successfully accepted.

Finally, let us consider that the channel $C_{4}$ is assigned to the user 0 . At the time $t=t_{4}$, the user 0 handovers to spotbeam $L$, and the user 4 still resides in the spot-beam $F$, and is using the channel $C_{4}$. Since the two cell $L$ and $F$ is distant less than the reuse distance from each other, the channel $C_{4}$ can not remain associate with the user 0 , if the user 4 is still active, that is, the user 0 should be assigned an channel different from the channel $C_{4}$. If there is no channel available for the cell $L$ at the time $t=t_{4}$, the user 0 is forced to terminate. Therefore, we cannot guarantee a successful handover to the user 0 .

In this example, only assigning the channel $C_{3}$ to the user 0 can provide all users with guaranteed handovers. Since the user locations are known and spot-beam movement is deterministic, the occurrence times of the handover events are available. Moreover, this availability makes us be able to assign channels to new calls to guarantee successful handovers to all calls. So far we have examined the basic principle of our new DCA algorithm. From now on, we describe our new DCA algorithm more precisely.

First, let's define $B_{i}(t)$ as the spot-beam that the user $i$ is expected to reside in at the time $t$ in future. The user $i$ can be an existing user or a new user who are requesting a new connection. Both the deterministic spot-beam movement and the user location information make us be able to define this precisely. Assuming that the two function $B_{i}(t)$ and $B_{j}(t)$ for two user $i$ and $j$ satisfy the following condition:

The cell $B_{i}(t)$ is distant not less than the reuse distance $D$ from the cell $B_{j}(t)$ for all $t \in\left[t_{0}, t_{\max }\right]$.

Where $t_{0}$ means the current time, and $t_{\max }$ is the minimum of the times for the two user $i$ and $j$ to depart from the footprint of the satellite in question. Then, the user $i$ and $j$ can use the same channel while they are under the footprint of the satellite in question. Assume that at the time $t=t_{0}$ a user terminal, which is denoted "user $x$ ", requires a connection to be setup. Let us denote with $\Lambda(x)$ as follows:

$$
\left\{c \mid \begin{array}{l}
\text { User } i \text { and } x \text { satisfies the above condition } \\
\text { for all user } i \in U(c) .
\end{array}\right\} .
$$

Where $U(c)$ denotes the set of users who use the channel $c$. If the user $x$ is assigned a channel $c \in \Lambda(x)$, we can guarantee the success of each handover of all of the users including the user $x$ from the definition. If the set $\Lambda(x)$ has no channel, the call setup request of the user $\mathrm{x}$ is rejected, that is, the call is blocked. If the set $\Lambda(x)$ has multiple channels, we choose and assign to the user $x$ a channel by the following criterion:

$$
\begin{aligned}
& \text { Channel } c^{*} \text { is allocated to the user } x \text {, if } \\
& d_{c^{*}}(x)=\min _{c \in \Lambda(x)}\left\{d_{c}(x)\right\}, \text { where } d_{c}(x) \text { is } \\
& \min _{i \in U(c)}\{\text { geographical distance between the user } x \text { and } i\} .
\end{aligned}
$$

The above criterion means that our DCA algorithm assigns to the user $x$ the channel in $\Lambda(x)$ that is being used by the user nearest to the user $x$. This channel selection criterion makes the channel repetition interval shorter. This is very important considering that the shorter the channel repetition interval, the greater the channel reuse over the whole system area.

We have introduced a new DCA algorithm to guarantee the success of each handover. This algorithm makes use of both the deterministic spot-beam movement and the user location information. It is worth to note that the Nearest Neighbor +1 strategy $(\mathrm{NN}+1)$ in [2] also guarantee the success of each handover, if all the spot-beams are hexagonal regular. The $\mathrm{NN}+1$ strategy selects the channel that is being used in the nearest cell at a distance of the reuse distance $D+$ 1 or more. In this paper, it is assumed that the call is rejected, if no channel satisfies this condition. (Conventional $\mathrm{NN}+1$ strategy selects a channel occupied in a cell at the distance of $D$, and then this cannot guarantee the success of each handover.) It is obvious that the $\mathrm{NN}+1$ strategy assigns to a new user $x$ a channel that is in $\Lambda(x)$, and hence provides zero call forced termination probability like our DCA algorithm. However, our algorithm provides a better performance than the $\mathrm{NN}+1$ strategy with respect to new call blocking probability. This will be shown in the next section.

\section{COMPUTATIONAL EXPERIMENTS}

In this section, we evaluate our new DCA algorithm through some computational experiments. The performance of the algorithm has been investigated using an event-driven computer simulation. The satellite used in our experiment has 100 spot-beams, which are assumed to have hexagonal regular cellular layout.

We use a simple model to obtain the traffic requirements that drive the experiment. First, we divide the earth surface by $1^{\circ}$ along with the latitude and longitude. Let $\pi_{m}$ to be the population of the square region $\mathrm{m}$, then we define the new call arrival rate for the square region $\mathrm{m}$ : 


$$
\lambda^{m}=M \times(\text { mean arrival rate }) \times \frac{\pi_{m}}{\sum_{i} \pi_{i}}
$$

Where $\mathrm{M}$ denotes the total number of the square regions on the earth surface. It is assumed that both the interarrival and call duration time are exponentially distributed.

The Nearest Neighbor (NN) scheme, the Nearest Neighbor $+1(\mathrm{NN}+1)$ scheme, and our new DCA algorithm are used and compared in our computational experiments. The NN strategy assigns an available channel that is being used in the nearest cell at a distance of D or more. It is known that the $\mathrm{NN}$ strategy has the lowest blocking rate under light traffic [2]. The Nearest Neighbor $+1(\mathrm{NN}+1)$ scheme was briefly introduced in the previous section. The forced call termination probability of this scheme is less than the NN strategy because the mobile terminal is likely to keep the present channel when moving into an adjacent cell. We already have mentioned that this strategy can provide zero call forced termination probability in LEO-MSS like our DCA algorithm in the previous section.

Fig. 2 shows the new call blocking probabilities for these three DCA scheme. The mean call duration time is assumed to be 120 seconds. The NN strategy has the best performance with respect to the new call blocking probability. And the $\mathrm{NN}+1$ strategy has the worst performance. This means that our algorithm is better than the $\mathrm{NN}+1$ strategy, since the two DCA scheme have the same performance with respect to forced call termination. The performances of these DCA schemes with respect to the handover dropping probability are shown in Fig. 3. As mentioned earlier, our DCA algorithm and the $\mathrm{NN}+1$ strategy provides zero forced call termination probability. It is interesting that the handover dropping probability of the $\mathrm{NN}$ strategy is lower than the new call blocking probability of this, even though this strategy doesn't prioritized the handover calls. This is due to the fact that an available channel for a cell is likely available for the adjacent cell, since the two cells have a lot of common interfering cells. Fig. 4 shows the completed call ratios for these DCA schemes. The completed call ratio is defined as the following and is closely related with the throughput of the system.

$$
\text { completed call ratio }=1-\frac{\text { number of blocked or dropped calls }}{\text { total number of arrived calls }}
$$

Our DCA algorithm is comparable to the NN strategy with respect to the completed call ratio as shown in Fig. 4. The $\mathrm{NN}+1$ strategy has a good performance with our algorithm in respect of the handover dropping probability, however has an inferior completed call ratio in comparison with the $\mathrm{NN}$ strategy and our DCA algorithm. Finally, our scheme utilizes frequency spectrum efficiently, and can also provide users a high-quality premium service that guarantees the success of each handover procedure, called guaranteed handover service.

Fig. 5, 6, and 7 show the blocking probability, dropping probability, and completed call ratio when the mean call duration time is set to 240 seconds. The results are similar to that in Fig. 2, 3, and 4. An interesting point is that the performance of our algorithm is slightly better that the NN strategy in respect of the completed call ratio as opposed to the case that the mean call duration time is 120 seconds. This shows that the more frequent handovers occur, the NN strategy produce the worse performance under the same traffic load. In other words, long mean call duration time or small spot-beam radius drives to use our algorithm.

\section{CONCLUSIONS}

In this paper, we suggest a new DCA scheme which eliminates forced call terminations due to handover failure (call dropping). This is based on position measurement and the characteristic of an LEO that the mobility in the system can be approximated by the deterministic movement of the satellite travel with a constant speed. The user location information and deterministic mobility model provide us the future handover behavior of a user terminal. The Nearest Neighbor $(\mathrm{NN})$ scheme, the Nearest Neighbor $+1(\mathrm{NN}+1)$ scheme, and our new DCA algorithm are used and compared in our computational experiments. The results of the computational experiments have shown that the completed call ratio of our algorithm is comparable with that of the NN strategy while our algorithm guarantees the success of each handover. Also, our scheme uses spectrum more efficiently than $\mathrm{NN}+1$ scheme, while providing guaranteed handover.

\section{REFERENCES}

[1] E. Del Re, R. Fantacci, and G. Giambene, "Efficient dynamic channel allocation techniques with handover queuing for mobile satellite networks," IEEE J. Select. Areas Commun., Vol. 13, pp. 397-405, Feb. 1995.

[2] Y. F. Hu, R. E. Sheriff, E. D. Re, R. Fantacci, and G. Giambene, "Satellite-UMTS traffic dimensioning and resource management technique analysis," IEEE Trans. Veh. Technol. Vol. 47, no. 4, pp. 13291341, 1998.

[3] M. M. -L. Cheng and J. C. -I. Chuang, "Performance Evaluation of Distributed Measurement-Based Dynamic Chanel Assignment in Local Wireless Communications," IEEE Journal on Selected Areas in Communications, Vol. 14, No. 4, May 1996.

[4] E. Del Re, R. Fantacci, and G. Giambene, "Handover Queuing Strategies with Dynamic and Fixed Channel Allocation Techniques in Low Earth Orbit Mobile Satellite Systems," IEEE Trans. Commun. Vol. 47, no. 1, pp. 89-102, Jan. 1999.

[5] M. Chiu and M. A. Bassiouni, " Predictive schemes for handoff prioritization in cellular networks based on mobile positioning," IEEE J. Select. Areas Commun., Vol. 18, no. 3, pp. 397-405, Mar. 2000.

[6] G. Maral, J. Restrepo, E. D. Re, R. Fantacci, and G. Giambene, "Performance analysis for a guaranteed handover service in an LEO constellation with a satellite-fixed-cell system," IEEE Trans. Veh. Technol. Vol. 47, no. 4, pp. 1200-1214, 1998.

[7] E. D. Re, R. Fantacci, and G. Giambene, "Different queuing policies for handover requests in low earth orbit mobile satellite systems," IEEE Trans. Veh. Technol. Vol. 48, no. 2, pp. 448-458, 1999.

[8] V. Obradovic and S. Cigoj, "Performance evaluation of prioritized handover management for LEO mobile satellite systems with dynamic channel assignment," IEEE GLOBECOM '99, pp. 296-300.

[9] H. Uzunalioglu, J. W. Evans, and J. Gowens, "A connection admission control algorithm for low Earth orbit satellite networks," Proc. ICC '99, pp. 1074-1078.

[10] P. Carter and M. A. Beach, "Evaluation of Handover Mechanisms in Shadowed Low Earth Orbit Land Mobile Satellite Systems," International Journal of Satellite Communications, Vol. 13, 1995. 


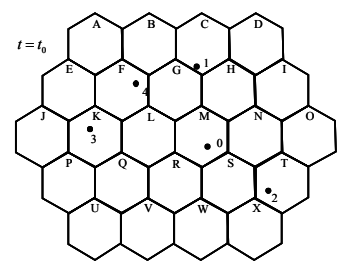

$V_{a b} \longrightarrow$

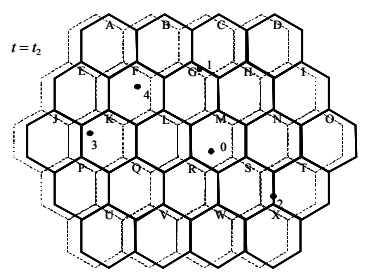

(a) Configuration $\mathrm{c}$

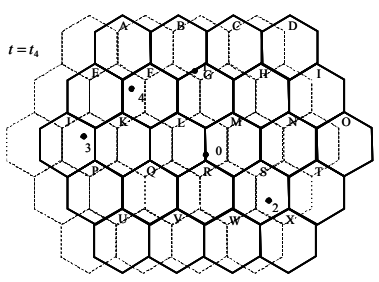

(a) Configuration e (a) Configuration a

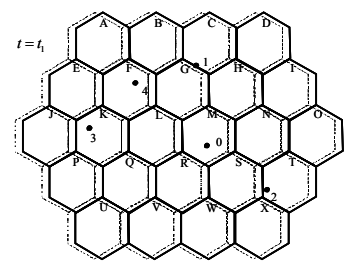

(b) Configuration $\mathrm{b}$

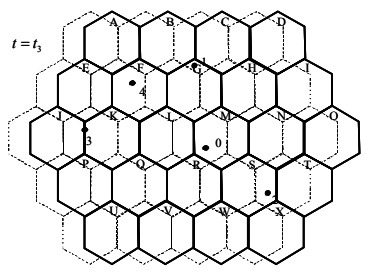

(b) Configuration $\mathrm{d}$

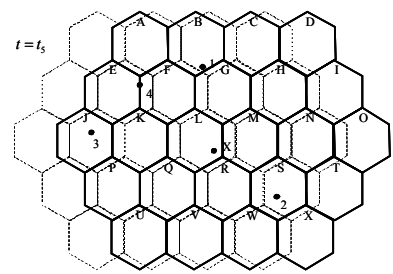

(b) Configuration $\mathrm{f}$

Fig. 1. An Example

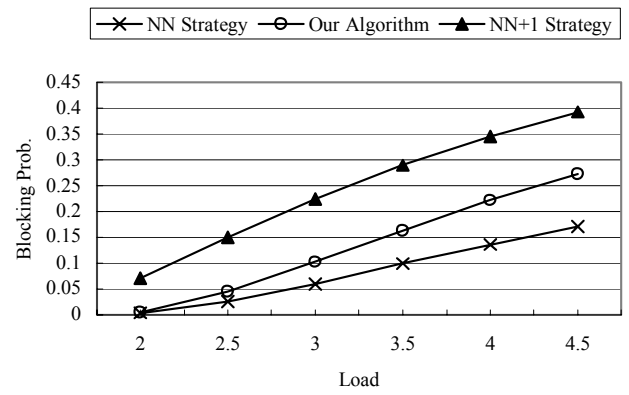

Fig. 2. Blocking Probability over Traffic Load (Mean Call Duration Time $=$ 120 seconds)

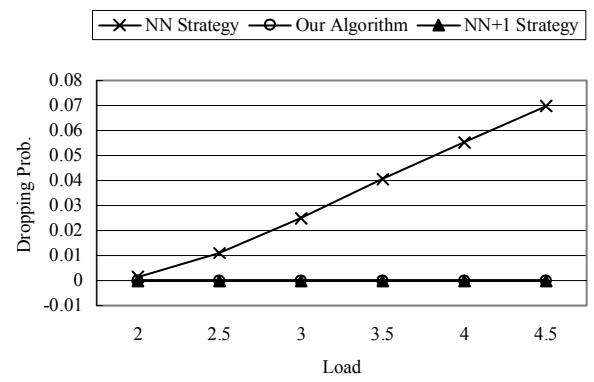

Fig. 3. Dropping Probability over Traffic Load (Mean Call Duration Time $=$ 120 seconds)

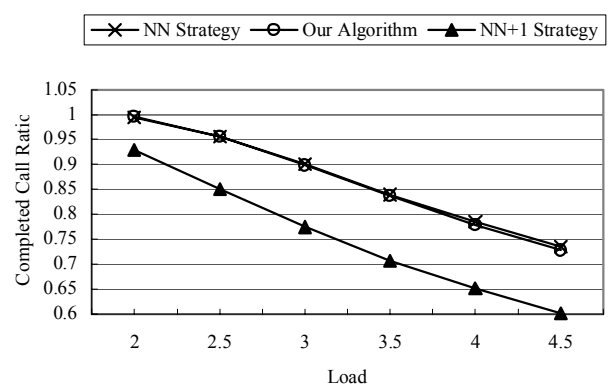

Fig. 4. Completed Call Ratio over Traffic Load (Mean Call Duration Time $=$ 120 seconds)

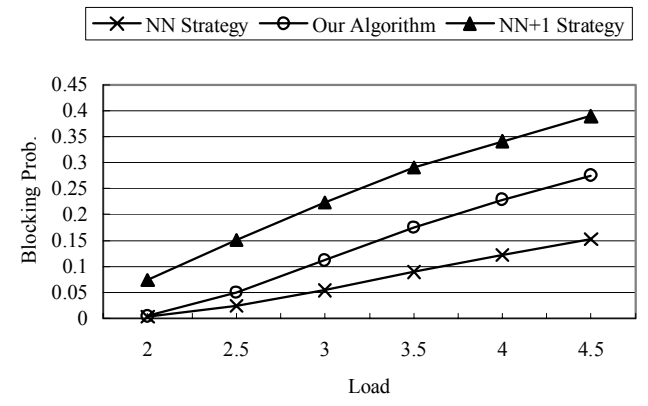

Fig. 5. Blocking Probability over Traffic Load (Mean Call Duration Time $=$ 240 seconds)

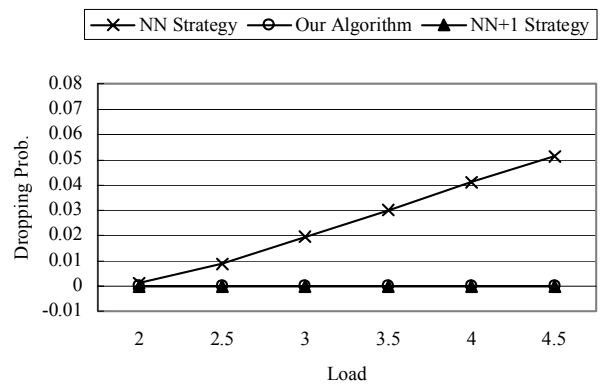

Fig. 6. Dropping Probability over Traffic Load (Mean Call Duration Time = 240 seconds)

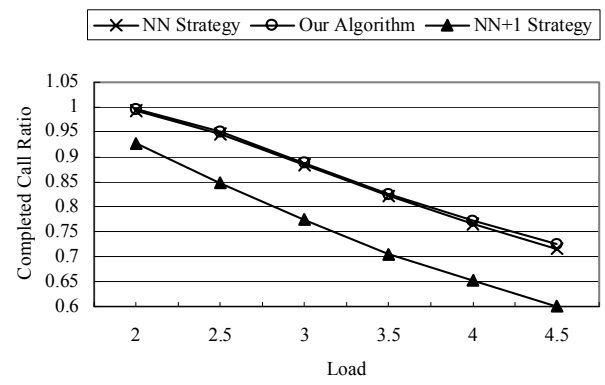

Fig. 7. Completed Call Ratio over Traffic Load (Mean Call Duration Time $=$ 240 seconds) 